\title{
Community acquired pneumonia incidence before and after proton pump inhibitor prescription: population based study
}

\author{
Fatmah Othman, ${ }^{1,2}$ Colin J Crooks, ${ }^{1}$ Timothy R Card ${ }^{1,3}$
}

Department of Epidemiology and Public Health, University of Nottingham, Nottingham NG5 1PB, UK

${ }^{2}$ Department of Basic Medical Sciences, King Saud bin

Abdulaziz University for Health

Sciences, Saudi Arabia

${ }^{3}$ NIHR Nottingham Digestive

Diseases Biomedical Research

Unit, Nottingham University

Hospitals NHS Trust and

University of Nottingham,

Nottingham, UK

Correspondence to: F Othman msxfo1@nottingham.ac.uk Additional material is published online only. To view please visit the journal online.

Cite this as: BMJ 2016;355:i5813 http://dx.doi.org/10.1136/bmj.i5813

Accepted: 18 October 2016

\section{ABSTRACT}

OBJECTIVE

To examine the risk of community acquired pneumonia before and after prescription of proton pump inhibitor (PPI) and assess whether unmeasured confounding explains this association.

DESIGN

Cohort study and self controlled case series.

SETTING

Clinical Practice Research Datalink (1990 to 2013) in UK. PARTICIPANTS

Adult patients with a new prescription for a PPI individually matched with controls.

\section{MAIN OUTCOME MEASURES}

Association of community acquired pneumonia with PPI prescription estimated by three methods: a multivariable Cox model comparing risk in PPI exposed patients with controls, corrected for potential confounders; a self controlled case series; and a prior event rate ratio (PERR) analysis over the 12 month periods before and after the first PPI prescription.

RESULTS

160000 new PPI users were examined. The adjusted Cox regression showed a risk of community acquired pneumonia 1.67 ( $95 \%$ confidence interval 1.55 to 1.79) times higher for patients exposed to PPI than for controls. In the self controlled case series, among $48451 \mathrm{PPI}$ exposed patients with a record of community acquired pneumonia, the incidence rate ratio was 1.19 (95\% confidence interval 1.14 to 1.25 ) in the 30 days after PPI prescription but was higher in the 30 days before a PPI prescription (1.92, 1.84 to 2.00). The Cox regressions for prior event rate ratio similarly showed a greater increase in community acquired pneumonia in the year before than the year after PPI prescription, such that the analysis showed a reduced relative risk of pneumonia associated with PPI use (prior event rate ratio $0.91,95 \%$ confidence interval 0.83 to 0.99 ).

\section{WHAT IS ALREADY KNOWN ON THIS TOPIC}

Studies have suggested that patients who are treated with proton pump inhibitors (PPIs) have an increased risk of developing community acquired pneumonia Potential confounders and bias may impair the interpretation of the association that has been observed in these studies

\section{WHAT THIS STUDY ADDS}

The crude association between PPI prescriptions and an increased rate in community acquired pneumonia could be explained by an underlying increased risk of pneumonia The confounding factors present before PPI use, rather than PPI use itself, were the main contributors to an increased rate of community acquired pneumonia in patients receiving PPIs

\section{CONCLUSION}

The association between the use of PPIs and risk of community acquired pneumonia is likely to be due entirely to confounding factors.

\section{Introduction}

Proton pump inhibitors (PPIs) have become the cornerstone of medical treatment for acid related gastrointestinal disorders. The widespread use of PPI treatment in clinical settings means that even if the health risks associated with their use are modest, they could have a substantial effect on a large number of patients. ${ }^{12}$ One of the adverse outcomes associated with PPI use that has attracted a considerable amount of attention in existing research is the possible increase in the risk of pneumonia. ${ }^{3-7}$ This association has been hypothesised to be related to bacterial overgrowth and colonisation as a result of alteration of the acidity of the stomach. ${ }^{45}$ Meta-analyses of randomised controlled trials have shown that the use of PPIs to prevent stress ulcers may increase the risk of hospital acquired pneumonia in critically ill patients. ${ }^{89}$ However, in the context of community acquired pneumonia, which is a common cause of morbidity and mortality especially among older people and those with other medical comorbidities, ${ }^{10}$ this link has been inconsistent in previous observational studies. ${ }^{11-16}$ These studies have been criticised for a lack of control for unmeasured confounding (such as patients' characteristics and comorbidities) $)^{17}$ and bias (such as confounding by indication) ${ }^{18}$ that may impair the significance of the association.

In the absence of data from randomised controlled trials, the variation in the risk of community acquired pneumonia among PPI users compared with non-users in previous studies may be attributable to differences between the groups in known and unknown risk factors. Advances in analytical techniques may enhance the validity of the results of observational studies by attempting to mitigate some of the effects of hidden confounding. We therefore aimed to assess whether confounding explains the association between the use of PPIs and the risk of community acquired pneumonia by using appropriate analytical techniques on observational data.

\section{Methods}

\section{Data source}

We used the Clinical Practice Research Datalink (CPRD), a large UK based electronic database of primary care records (www.cprd.com). ${ }^{19}$ CPRD has been extensively used and validated for pharmacoepidemiology research. ${ }^{19-21}$ The database contains anonymised information about patients that encompasses medical diagnoses, prescriptions, investigations, and referrals to 
secondary care. More than $50 \%$ of the practices that include their records in the CPRD have consented to their patients' records being linked with Hospital Episode Statistics data and Office for National Statistics mortality data. ${ }^{19}$ Hospital Episode Statistics data contain information on all patients who have been admitted to hospital, together with information about the primary and secondary causes of each episode of inpatient care (with diagnosis coded using ICD-10 (international classification of diseases, 10th revision)), type of admission, procedure performed, length of stay, and discharge status. ${ }^{22}$

\section{Study population}

The study population included people aged 18 years or over and registered in the CPRD from 1 January 1990 to 1 August 2013. Patients had to have at least one year of registration on CPRD after the later of the date of current registration and the date the practice became "up to standard" on CPRD, ${ }^{21}$ as well as an "acceptable" registration status as defined by CPRD. ${ }^{21}$ We identified an exposed cohort from this source population as a random sample of 160000 people who received a first prescription for a PPI in CPRD at least one year after they started contributing data during the study period (that is, new users). We calculated the duration of each PPI prescription by using the number of treatment days recorded by the general practitioner or dividing the total prescription quantity by the numeric daily dose prescribed for each prescription.

We also identified a subset of patients with linked Hospital Episode Statistics/Office for National Statistics data within the study population and selected these patients to improve the ascertainment of the outcome of this study (that is, community acquired pneumonia). For this subset, we looked specifically at patients with more severe community acquired pneumonia requiring hospital admission by limiting the definition of pneumonia to those coded within Hospital Episode Statistics/Office for National Statistics data. We also modified the follow-up period to be consistent with the linkage coverage period (from January 1998 to January 2012).

\section{Outcome}

We defined community acquired pneumonia in the CPRD by the presence of a Read code for this diagnosis (list of codes available on request). The list included codes for chest infection and lower respiratory tract infection to ensure that all cases of community acquired pneumonia categorised in this way were captured. We refer to this as our broad primary care definition and also used a narrow primary care definition eliminating codes not specific for pneumonia. We regarded pneumonia diagnoses recorded less than 84 days apart as the same event, as we considered 84 days to be sufficient for the clinical condition and lung function to have returned to the baseline state. ${ }^{2324}$ For patients with Hospital Episode Statistics/Office for National Statistics linked data, we identified episodes of hospital admission that were allocated a primary diagnosis of community acquired pneumonia or a death certificate that was issued between March 1997 and April 2012 on which pneumonia was listed as a contributing or underlying cause of death (on the basis of ICD coding).

\section{Study design}

We used self controlled case series and cohort study designs in this study.

\section{Self controlled case series study}

The self controlled case series analysis method is a type of cohort study in which relative risk is based on within person comparisons between exposed and unexposed observation time, so only exposed patients with events can be included. ${ }^{25}$ The advantage of this design is that the influence of between person confounding will be eliminated. ${ }^{2526}$ From the cohort exposed to PPIs, we selected all the cases with a recorded diagnosis of community acquired pneumonia. We considered the exposure to PPIs to begin on the date of PPI prescription and to end after its calculated duration including any consecutive prescriptions, plus an additional 30 day washout period. We then divided the observation time into risk windows for each person: 30 days before starting the first PPI prescription; 0-30 days after starting the first PPI prescription; and the remaining exposed period with the post-exposure 30 day period. The baseline (unexposed) period consisted of all observation times for which no PPI prescription was issued within the study period (fig 1). The purpose of the additional 30 day period before the first prescription was to assess whether any increase in community acquired pneumonia rate was associated with an acute event that might have precipitated the PPI prescription.

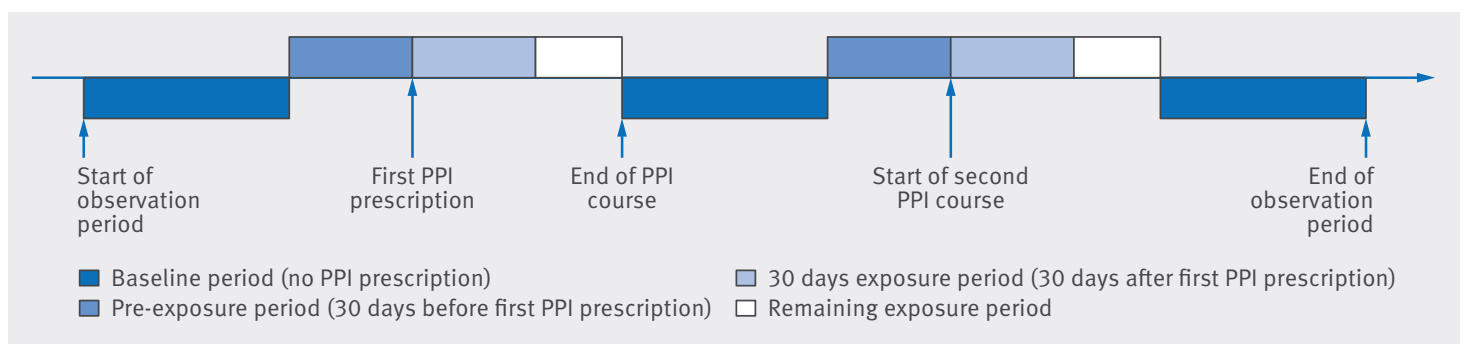

Fig 1 | Illustration of self controlled case series method showing division of time of each person included in this analysis to assess incidence of community acquired pneumonia in relation to prescription for proton pump inhibitor (PPI). Events of pneumonia can occur during baseline or exposure period 


\section{Cohort study}

For this study design, we individually matched the exposed patients one to one by age (within 5 years), sex, and year of prescription with an unexposed cohort who had never received a PPI prescription. We required these unexposed controls also to have been contributing data for at least one year before their matched patient's first prescription. The following potential confounders were extracted and evaluated from the CPRD primary care record, both for all CPRD patients and for the subset of patients with Hospital Episode Statistics/Office for National Statistics linked data: smoking status (categorised into smoker, never smoked, or missing), alcohol use (never drinker, ever drinker, or missing), number of visits to the general practitioner in the year before the PPI prescription (five visits or fewer, or more than five visits), immunosuppression (defined as chemotherapy treatment within the previous six months, systemic high dose steroid treatment for three months, receipt of certain immunosuppressive drugs, having a solid organ transplant with any anti-rejection drugs in the previous year, or having a diagnosis of HIV infection ${ }^{27}$ ), and comorbidities categorised on the basis of the Charlson index score. ${ }^{28}$ We assessed comorbidities by using the entire patient record before the first PPI prescription date for the exposed cohort or the matched date for the unexposed cohort. We also included prescriptions of systemic corticosteroid and opioid (within 90 days before the prescription or matched date). In addition, we included data on the socioeconomic status measured using the Index of Multiple Deprivation 2007.

\section{Statistical analyses}

We calculated descriptive summary statistics for the study population by exposure status. We then did the analyses below, both for all CPRD patients and for the subset of patients with Hospital Episode Statistics/ Office for National Statistics linked data. For each analysis, we present results for our three different definitions of community acquired pneumonia (broad primary care definition, narrow primary care definition, and hospital and mortality records based definition). We used the same cohort across the study designs to avoid using multiple cohorts, because this may cause confusion when comparing the study results. We used Stata 12 for all statistical analyses.

Follow-up time

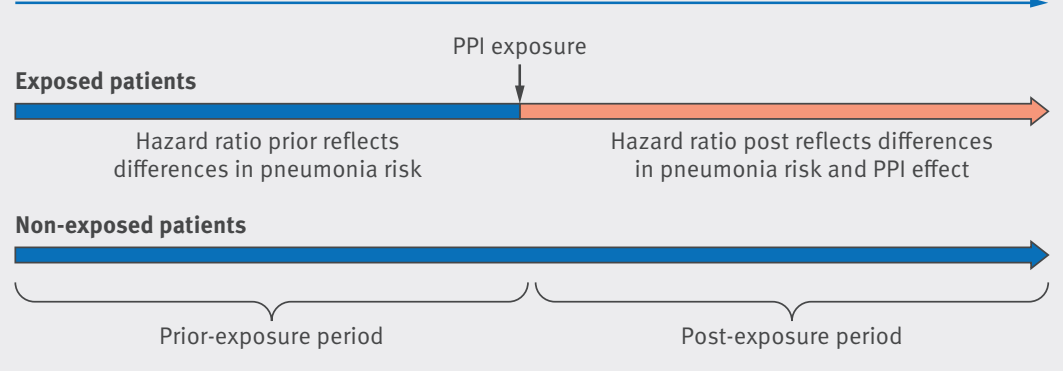

Fig $2 \mid$ Prior event rate ratio adjustment method. Prior period means time before index date (proton pump inhibitor (PPI) prescription date for exposed cohort and matched date for unexposed patients); post period means time after PPI exposure

\section{Self controlled case series analysis}

We used conditional Poisson regression models with fixed effects to calculate the incidence rate ratio comparing the incidence rate of community acquired pneumonia during the exposure period with the incidence rate during the baseline period, with adjustment for age (5 year bands). ${ }^{2526}$ This allows inclusion of multiple events of pneumonia during the observation period and adjusts for the correlations within individuals. Thus, the data were expanded to panel data, so that each row corresponded to a single time interval for each case. We then identified the numbers of events that occurred within each interval. We excluded person time after the pneumonia event until the day after the date of resolution of pneumonia, because no additional events could occur until the current pneumonia had resolved.

\section{Cohort study}

We did two analyses using this study design: a multivariable Cox proportional hazards analysis and a prior event rate ratio analysis. ${ }^{29-31}$

In the adjusted Cox regression analysis, we considered the first occurrence of community acquired pneumonia after the index date. We used Cox proportional hazards modelling to estimate the hazard ratio for pneumonia in people receiving PPIs, adjusted for potential confounders, with 95\% confidence intervals. We followed the cohorts from the date of their first PPI prescription until the earliest of the pneumonia event date, prescription end date, or study end date (this was the earliest date of the following: end of observation time (1 August 2013), date of death, date transferred to a different practice, or last date of data collection from practice). We tested the validity of the proportional hazards assumption by plotting log-minus-log survival curves and carrying out Schoenfeld tests. We included each potential confounder in the model if they modified the hazard ratio by more than $10 \%$. We categorised missing data into a separate category.

The prior event rate ratio adjustment method has recently been proposed as a means of reducing the bias that results from residual confounding. ${ }^{29-31}$ This method requires that both exposed and unexposed cohorts did not receive the study drug before the index date-that is, the date at which the exposed cohort first received the prescription and the matched date for the unexposed patients. The method relies on a before and after design and is based on the assumption that the differences in outcomes between exposed and unexposed patients before receiving the treatment reflect the combined effect of confounders independently of any effect of the study drug. ${ }^{2931}$ The prior event rate ratio is estimated by the ratio of two unadjusted hazard ratios: the unadjusted hazard ratio for community acquired pneumonia during the year after initial PPI prescription for the exposed group versus the unexposed group (HR.post) and the unadjusted hazard ratio for community acquired pneumonia before the PPI prescription of the exposed versus the unexposed people (HR.prior)-prior event rate ratio adjusted hazard ratio=HR.post/HR.prior (fig 2). Therefore, the prior event rate ratio provides an estimate of the effect the drug exposure had on the hazard adjusted for 
confounding. ${ }^{31}$ The follow-up of patients in the year after initial PPI prescription ran from the date of their first PPI prescription until the earliest of the anniversary of that date, the occurrence of a pneumonia event, or the study end date. For the year before the initial PPI prescription, patients were followed from the date one year before starting the PPI until the earliest of a pneumonia event or the start of the PPI. We obtained the 95\% confidence intervals of the prior event rate ratio by bootstrapping. ${ }^{2931}$

\section{Sensitivity analyses}

As with any statistical method, several assumptions must be met for both the self controlled case series analysis and prior event rate ratio analysis methods to be considered reliable. We did sensitivity analyses to assess the possibility that these assumptions had been violated. For the self controlled case series, the first assumption is that the occurrence of recurrent outcome events must be independent-that is, the occurrence of one event must not alter the probability of a subsequent event occurring. ${ }^{25}$ We did a sensitivity analysis restricted to the first event of community acquired pneumonia during the observation period to assess this assumption. The second assumption of the self controlled case series, as well as for prior event rate ratio, was that the occurrence of one event should not alter the probability of subsequent exposure. ${ }^{2531}$ Although pneumonia is not a recognised indication, caution, or contraindication for the use of PPIs, it is likely that hospital admission could lead to the prescription of a PPI. ${ }^{32}$ To account for this, we did an analysis that was limited to new users of PPIs who had not been admitted to hospital in the 60 days before the first PPI course. The third assumption is that mortality subsequent to an event should not alter the probability of subsequent exposure. ${ }^{2631}$ For the self controlled case series analysis, we did a sensitivity analysis that excluded people who had died within 90 days of the recorded community acquired pneumonia, as such cases could have used PPIs only before community acquired pneumonia and not afterwards. Similarly, for the prior event rate ratio analysis, we did sensitivity analysis restricted to non-fatal pneumonia on the basis that it would be impossible for fatal pneumonia cases to occur in the one year before the first PPI prescription, whereas fatal pneumonia could occur afterwards. Also, we carried out a sensitivity analysis that was limited to cases before the point at which the first large population based study suggesting an association was published in a specialised medical journal, ${ }^{11}$ to minimise the risk that knowledge of patients' PPI use could affect diagnosis of pneumonia or that previous pneumonia events could affect the physician's decision to prescribe a PPI.

For the Cox regression analysis and prior event rate ratio, we did a sensitivity analysis restricted to people over the age of 65 years (who are eligible to receive free prescriptions and therefore were less likely to purchase over the counter drugs) and to the time period before the availability of the over counter PPIs in the UK. ${ }^{33}$

\section{Sample size calculation}

We used the stpower Cox command to calculate the minimum number of events needed to detect a hazard ratio of 1.1 with $90 \%$ power and $\alpha=0.05$. With an estimated incidence rate of 0.6 per 100 unexposed person years, ${ }^{1123}$ we estimated that 160000 patients were needed assuming an average five year follow-up period for each patient and constant pneumonia risk over calendar time. This study population of 160000 PPI exposed patients, therefore, was randomly sampled from the whole CPRD population according to the power calculation outlined above.

\section{Patient involvement}

No patients were involved in the development of the research question or the outcome measures. No patients were involved in developing plans for recruitment, design, or implementation of the study, nor were they asked to advice on interpretation or writing up of results. There are no plans to disseminate the results of the research to study participants.

\section{Results}

We identified 160000 new users of PPIs during the study period who were matched individually to an equal number of non-PPI exposed patients. The mean age at entry to the study was 56 (SD 16) years for both exposed and unexposed cohorts, and 55\% (n=88455) of each cohort were women. Compared with unexposed patients, those prescribed PPIs were more likely to have a history of smoking $(42.9 \% v 33.7 \%$ of their matched controls) and alcohol use (29.1\% v 23.9\%); they also had a higher burden of comorbidity and used more corticosteroids and opioids (table 1). Hospital Episode Statistics/Office for National Statistics linked information was available for 257886 of the patients, whose characteristics were largely similar to those of the complete cohort (table 1). Most patients used PPIs for a short period, with a median duration of 28 (interquartile range 28-76) days.

\section{Cox regression analysis}

In this analysis, the exposed patients had an increased hazard of pneumonia defined by each of the three definitions of community acquired pneumonia. Hazard ratios were greater when we defined community acquired pneumonia more narrowly, with unadjusted hazard ratios of 5.44 (95\% confidence interval 4.23 to 6.99 ) and 4.76 (4.12 to 5.49) when we used narrow primary care definitions and hospital/mortality records based definitions, respectively (table 2). After adjustment, the hazard of pneumonia by our broad primary care definition fell to 1.67 (1.55 to 1.79) times that of controls in PPI exposed patients, and the hazard ratios were similarly reduced by adjustment for confounding when our narrower primary care definitions of pneumonia were used (table 2). In each case, however, we found a statistically significant excess of pneumonia in the exposed cohort. As the Charlson's index was developed to predict mortality and not morbidity, we carried out a separate analysis adjusted for individual important potential confounders for pneumonia that resulted in a slight reduction in the hazard ratio (table 2 ). 


\begin{tabular}{|c|c|c|c|c|}
\hline \multirow[b]{2}{*}{ Characteristic } & \multicolumn{2}{|c|}{$\begin{array}{l}\text { Complete cohorts } \\
(\mathrm{n}=320000)\end{array}$} & \multicolumn{2}{|c|}{$\begin{array}{l}\text { HES-ONS linked patients } \\
(\mathrm{n}=257886)\end{array}$} \\
\hline & $\begin{array}{l}\text { Unexposed } \\
(n=160000)\end{array}$ & $\begin{array}{l}\text { Exposed } \\
(n=160000)\end{array}$ & $\begin{array}{l}\text { Unexposed } \\
(n=128943)\end{array}$ & $\begin{array}{l}\text { Exposed } \\
(\mathrm{n}=128943)\end{array}$ \\
\hline \multicolumn{5}{|l|}{ Smoking: } \\
\hline Never smoked & $59581(37.2)$ & $57805(36.1)$ & $47243(36.7)$ & $45757(35.5)$ \\
\hline Smoker & $53889(33.7)$ & $68612(42.9)$ & $43633(33.8)$ & $55935(43.4)$ \\
\hline Missing & $46530(29.1)$ & $33583(21.0)$ & $38067(29.5)$ & $27251(21.1)$ \\
\hline \multicolumn{5}{|l|}{ Alcohol use: } \\
\hline Never drinker & $48961(30.6)$ & $51747(32.3)$ & $40394(31.3)$ & $42716(33.1)$ \\
\hline Ever drinker & $38292(23.9)$ & $46524(29.1)$ & $29364(22.8)$ & $36246(28.1)$ \\
\hline Missing & $72747(45.5)$ & $61729(38.6)$ & $59185(45.9)$ & $49981(38.8)$ \\
\hline \multicolumn{5}{|c|}{ Index of Multiple Deprivation fifths*: } \\
\hline Unavailable & $4833(3.0)$ & $4333(2.7)$ & $24(0.02)$ & $26(0.02)$ \\
\hline 1 (least deprived) & $41096(25.7)$ & $36149(22.6)$ & $34359(26.6)$ & $29987(23.3)$ \\
\hline 2 & $37827(23.6)$ & $36504(22.8)$ & $31588(24.5)$ & $30236(23.4)$ \\
\hline 3 & $30651(19.2)$ & $30904(19.3)$ & $25464(19.8)$ & $25739(20.0)$ \\
\hline 4 & $27332(17.1)$ & $30002(18.8)$ & $22542(17.5)$ & $24867(19.3)$ \\
\hline 5 (most deprived) & $18261(11.4)$ & $22108(13.8)$ & $14966(11.6)$ & $18088(14.0)$ \\
\hline \multicolumn{5}{|l|}{ Charlson comorbidity index score: } \\
\hline 0 & $99492(62.2)$ & $72290(45.2)$ & $79978(62.0)$ & $58048(45.0)$ \\
\hline $1-2$ & $48287(30.2)$ & $63461(39.7)$ & $39124(30.4)$ & $51098(39.6)$ \\
\hline $3-4$ & $9510(5.9)$ & $17494(10.9)$ & $7775(6.0)$ & $14413(11.2)$ \\
\hline$>5$ & $2711(1.7)$ & $6755(4.2)$ & $2066(1.6)$ & $5384(4.2)$ \\
\hline \multicolumn{5}{|l|}{ No of general practitioner visits: } \\
\hline$\leq 5$ & $87286(54.5)$ & $37605(23.5)$ & 69788 (54.1) & $29582(22.9)$ \\
\hline$>5$ & $72714(45.4)$ & $122395(76.5)$ & $59155(45.9)$ & $99361(77.1)$ \\
\hline Immunosuppressiont & $4882(3.0)$ & $16609(10.3)$ & $3866(3.0)$ & $13723(10.6)$ \\
\hline Non-topical corticosteroid use & $5832(3.6)$ & $15990(9.9)$ & $4814(3.7)$ & $13221(10.2)$ \\
\hline Opioid prescriptions & $1557(0.9)$ & $9469(5.9)$ & $1261(0.9)$ & $7809(6.0)$ \\
\hline
\end{tabular}

\section{Self controlled case series}

From the exposed cohort, we identified 48451 PPI exposed patients in CPRD with a record of at least one community acquired pneumonia (broad primary care definition), with a mean age of 60 (SD 16) years, and 5582 patients in Hospital Episode Statistics linked data who had a record of pneumonia related to hospital admission or death, with mean age of 70 (SD 14) years.

Table 3 shows the incidence rate ratios for the different risk windows and pneumonia definitions. Using our broad definition, the incidence rate ratio for pneumonia among PPI users was 1.19 (1.14 to 1.25) for 30 days after PPI prescription and 1.49 (1.46 to 1.52) for the remaining exposure period compared with the baseline period (table 3). The incidence rate ratio for the 30 days before exposure was even higher at 1.92 (1.84 to 2.00). As for the Cox regression, differences in ratios were higher for the more narrow definition of pneumonia (table 3).

\section{Prior event rate ratio analysis}

The rate of pneumonia for the exposed patients was similar before a PPI prescription (62.1 per 1000 person years of follow-up) to the rate after a PPI prescription (61.4 per 1000 person years of follow-up), whereas the rate of pneumonia in unexposed patients increased over the study period (table 4 ). The prior event rate

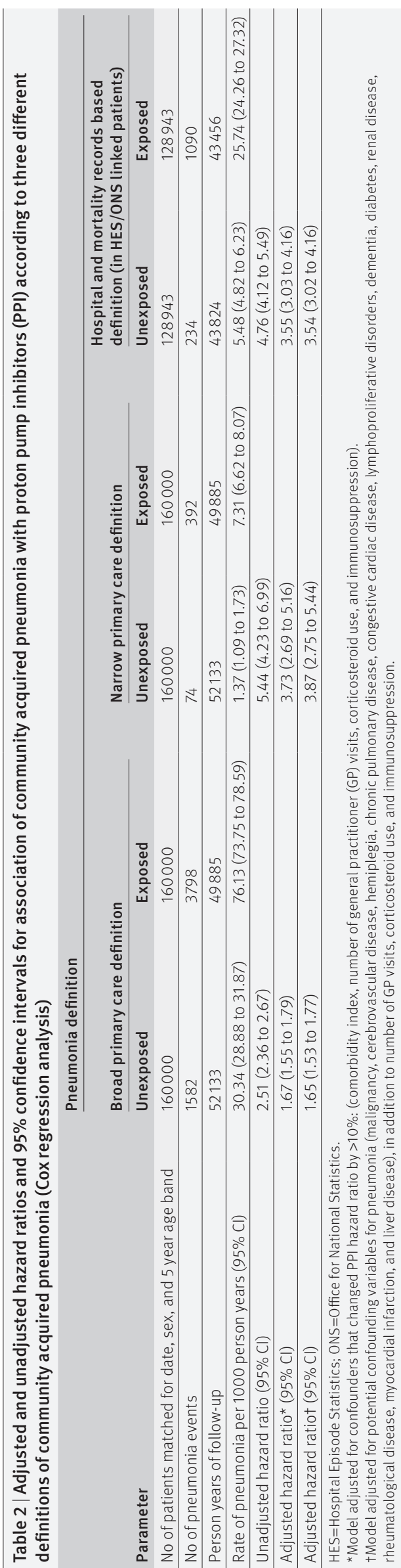



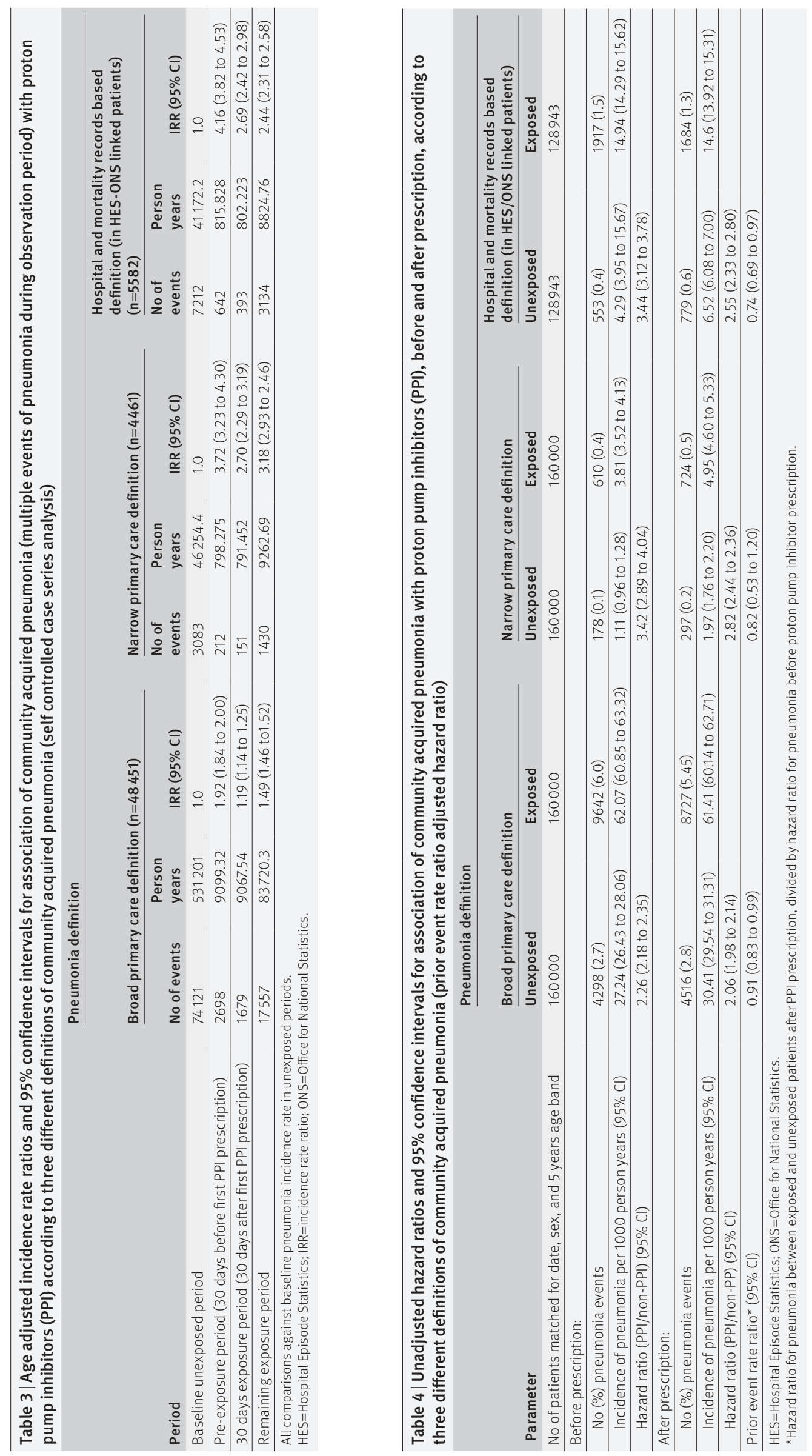

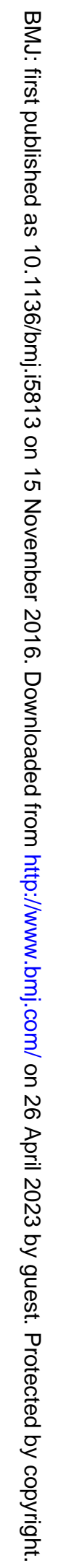


ratio for pneumonia (broad primary care definition) was 0.91 (0.83 to 0.99 ) on the basis of an unadjusted post-PPI hazard ratio of 2.06 (1.98 to 2.14) divided by an unadjusted prior-PPI hazard ratio of 2.26 (2.18 to 2.35). Similarly, the prior event rate ratio for pneumonia in Hospital Episode Statistics/Office for National Statistics subset was 0.74 (0.69 to 0.97) (table 4).

\section{Sensitivity analysis}

For the self controlled case series analysis, restricting the primary analysis to only the first event recorded resulted in reductions in the observed rate ratios of pneumonia after PPI prescriptions, whereas no change was seen in the risk of pneumonia before the PPI prescription (supplementary table A). After exclusion of the $20 \%$ of patients in the self controlled case series analysis who had been admitted to hospital before their first PPI prescription, the incidence rate ratio for community acquired pneumonia (using our broad primary care definition of pneumonia) was very little changed from that of our primary analysis (supplementary table B). Around 5\% of patients died within 90 days of the date of diagnosis of pneumonia, and the results of the analysis excluding these patients showed a reduction in the incidence rate ratio for pneumonia for 30 days after PPI prescription and for the remaining exposure period (supplementary table C).

For the prior event rate ratio method, both of the analyses to test for a possible violation of the underlying assumption concerning the effect of hospital admission on the probability of subsequent PPI exposure and the effect of mortality produced similar results to the primary analysis (supplementary tables D and E). The results from the remaining sensitivity analyses were very similar to those from the whole population for Cox regression and prior event rate ratio analyses (supplementary tables F-J).

\section{Discussion}

This study has shown that a crude association exists between PPI prescriptions and an increased rate of community acquired pneumonia, but that this is explained by an underlying increased risk of pneumonia in patients preceding a PPI prescription. Furthermore, after adjustment for confounding and changes over time, the relative rate of community acquired pneumonia actually fell after PPI prescriptions.

\section{Strengths and limitations of study}

A major strength of this study is that we have used routinely collected general practice records to conduct it. This provides for an unbiased selection of both the exposed and control cohorts and reduces the opportunity for information bias (as both PPI prescription and pneumonia diagnosis were prospectively collected independently). In addition, as the base population is representative of the UK general population, our results are likely to be generalisable at least to the UK population and to other similar populations. ${ }^{21}$ However, the generalisability of self controlled case series analysis is limited, as patients who are included may have a different underlying risk of community acquired pneumonia than the baseline population.

A further strength is that data were available relating to several potential confounders, allowing us to control for the effects of these in the Cox regression models. However, missing data with respect to smoking and alcohol use show a risk of residual confounding by these factors in addition to unmeasured confounding. An important methodological strength therefore is that we have used two analytical methods to mitigate the effect of confounders that are difficult to measure or incompletely measured.

We used the same patients as their own control in the case series method to minimise confounding due to patients' characteristics and comorbidities. The conditional Poisson regression model represented an effective choice in our analysis of recurrent events of community acquired pneumonia because previous studies have shown it to be effective in terms of the over-dispersion and autocorrelation of panel data. ${ }^{34} 35$ Also, we examined the key assumptions underlying the self controlled case series method by looking for evidence that the occurrence of pneumonia does not alter the risk of subsequent PPI prescription. Excluding from the main analyses those patients who had been admitted to hospital or died after pneumonia events had no substantial effect on the results. However, as this method adjusts for a fixed relative difference in confounders between the exposed and unexposed cohorts, it does not reduce confounding due to factors that may vary over time differently between the exposed and unexposed people.

We also attempted to overcome the limitations of previous observational work by using a newer analytical technique, prior event rate ratio, which has been proposed as a method of controlling for unmeasured time-fixed confounding. ${ }^{29} 31$ With this method, we were able to adjust both for the underlying increased risk of pneumonia in patients who were treated with PPIs (that confounded a standard Cox regression) and for changes in pneumonia incidence over time (that may have confounded the case series analysis). Although prior event rate ratio methods offer a promising approach to overcome biases that can arise in observational studies, in practice we cannot exclude all situations in which previous events might influence future treatments. However, we attempted to assess the potential biases carefully by examining the possible causes of violations of the prior event rate ratio assumptions via either hospital admission, death, or the publication of studies of pneumonia risks influencing PPI prescriptions, and we found no evidence to suggest that prior event rate ratio represented an inappropriate analytical technique in our study.

One potential limitation is the possibility of error in our assessment of exposure, as CPRD data includes only information about the prescriptions and does not prove that the prescribed drug has been taken. This could lead to exposure misclassification, especially in the case of patients who took PPI intermittently. However, as PPIs are initially prescribed for symptom 
control, this may not lead to a large overestimation. Additionally, previous studies reported that relying on prescription information as a surrogate for PPI use is unlikely to introduce significant misclassification. ${ }^{14}$

Although the information contained in the CPRD has been validated for a wide range of diagnoses, including other respiratory disease ${ }^{36}$-and in particular the diagnosis of pneumonia in the linked CPRD-Hospital Episode Statistics data has been examined ${ }^{10}$ - general practitioners often diagnose community acquired pneumonia without a radiograph, which may lead to misclassification between this and other conditions (for example, chest infection or chronic respiratory tract infection). We tried to reduce the effect of such misclassification by using more specific diagnostic codes for community acquired pneumonia (using our narrow definition) and by an analysis limited to patients with hospital or death certificate diagnoses in the sensitivity analyses.

\section{Comparison with existing literature}

Many studies have examined the association between PPI treatment and the risk of community acquired pneumonia. Early observational studies showed that using PPIs might be associated with an increased risk of community acquired pneumonia. ${ }^{11-13}$ Further studies suggested that a dose-response relation supported causality of the association, ${ }^{116}$ and yet others argued that a temporarily increased risk suggested the presence of protopathic bias. ${ }^{17} 18$ There have also been studies including a retrospective analysis of the original safety data from several randomised clinical trials of esomeprazole (one specific PPI), ${ }^{37}$ which failed to show any such association despite statistical adjustment for confounders. ${ }^{1415}$ Concern was amplified, however, by findings from meta-analyses based on these observational studies that showed that the risk of community acquired pneumonia was 34-49\% higher among patients who used PPIs than it was among non-users, with a temporal relation soon after the start of the PPI treatment. $^{57}$ Thus, evidence linking PPI treatment to the risk of community acquired pneumonia remains inconclusive.

We have examined the association of PPI use and community acquired pneumonia and assessed whether residual and time-fixed confounding explains this association. The results of the adjusted Cox regression model in this study did not differ from either the early observational studies or previous studies that used CPRD data and found a similar association. However, self controlled case series analysis shows that this risk predates prescription, and the prior event rate ratio analysis suggests that the significant association observed can be attributed to confounding. Previous studies have reported that the highest risk of pneumonia occurs in the first 30 days, whereas we observed an incidence rate ratio of 1.19 in the 30 days after PPI prescription and 1.49 for the remaining exposure period. This apparent contradiction may be explained by differences in the subpopulations of patients taking long term and short term PPIs; as most courses in our data were short term, the initial 30 days will primarily reflect these patients whereas the later period will be long term users.

Our findings from the prior event rate ratio analysis are comparable to those of a recent study that analysed the pooled adverse event data related to respiratory tract infection reported in randomised double blind clinical studies that were specific to esomeprazole. ${ }^{38}$ The reported relative risk for pneumonia in patients receiving esomeprazole compared with the placebo in that study was 0.66 (95\% confidence interval 0.36 to 1.22). ${ }^{38}$ Although the populations recruited to randomised controlled trials are often not typical of those receiving PPIs in general practice, our results suggest that these findings may well be widely generalisable. We believe that this comparison is also an important validation of the prior event rate ratio method, which has had limited use previously.

\section{Conclusion}

Our results indicate that no strong evidence exists to support the association between the use of PPIs and an increase in the risk of community acquired pneumonia. Patients who are treated with PPIs have an underlying risk of community acquired pneumonia before receiving the PPI prescription. Therefore, patients' characteristics, comorbidity, and severity of gastro-oesophageal reflex disease were probably the main contributors to the increased risk of pneumonia observed in patients who received PPIs. ${ }^{1718}$

Contributors: TRC proposed the original idea for the study, planned the study design and the analysis, was involved in interpretation of results, and revised the paper critically. FO helped in planning the analysis and was responsible for data management, statistical analyses, and writing the first draft of the paper. CJC contributed to study design and concept, analysis planning, and interpretation of results, as well as to revising the drafts of the paper. All authors approved the final version. FO is the guarantor.

Funding: FO carried out this study as part of her PhD programme at the University of Nottingham. She is a staff member at King Saud bin Abdulaziz University for Health Sciences (KSAU-HS), Saudi Arabia. She has received a scholarship award from KSAU-HS, which sponsors her studies at University of Nottingham. The KSAU-HS had no role in the design, analysis, data interpretation, or preparation of this manuscript. TRC and CJC were independent of the funder.

Competing interests: All authors have completed the ICMJE uniform disclosure form at www.icmje.org/coi_disclosure.pdf (available on request from the corresponding author) and declare that: FO has received a scholarship award from King Saud bin Abdulaziz University for Health Sciences, Saudi Arabia, which sponsors her studies at the University of Nottingham; no financial relationships with any organisations that might have an interest in the submitted work in the previous three years; no other relationships or activities that could appear to have influenced the submitted work.

Ethical approval: This study was approved by the Independent Scientific Advisory Committee (ISAC) with CPRD number 13_215.

Data sharing: CPRD data were provided under a licence that does not permit sharing. Data are, however, obtainable directly from CPRD under their standard conditions.

Transparency: The lead author affirms that the manuscript is an honest, accurate, and transparent account of the study being reported; that no important aspects of the study have been omitted; and that any discrepancies from the study as planned (and, if relevant, registered) have been explained

This is an Open Access article distributed in accordance with the Creative Commons Attribution Non Commercial (CC BY-NC 3.0) license, which permits others to distribute, remix, adapt, build upon this work non-commercially, and license their derivative works on different terms, provided the original work is properly cited and the use is noncommercial. See: http://creativecommons.org/licenses/by-nc/3.0/. 
1 Mazer-Amirshahi M, Mullins PM, van den Anker J, Meltzer A, Pines $J M$. Rising rates of proton pump inhibitor prescribing in US emergency departments. Am J Emerg Med 2014:32:618-22. doi:10.1016/j.ajem.2014.03.019.

2 Cahir C, Fahey T, Tilson L, Teljeur C, Bennett K. Proton pump inhibitors: potential cost reductions by applying prescribing guidelines. $B M C$ Health Serv Res 2012;12:408. doi:10.1186/1472-6963-12-408.

3 Heidelbaugh JJ, Goldberg KLIJ. Adverse risks associated with proton pump inhibitors: a systematic review. Gastroenterol Hepatol 2009;5:725-34

4 Fohl AL, Regal RE. Proton pump inhibitor-associated pneumonia: Not a breath of fresh air after all?World J Gastrointest Pharmacol Ther 2011;2:17-26. doi:10.4292/wjgpt.v2.i3.17.

5 Eom C-S, Jeon CY, Lim J-W, Cho E-G, Park SM, Lee K-S. Use of acid-suppressive drugs and risk of pneumonia: a systematic review and meta-analysis. CMAJ 2011;183:310-9. doi:10.1503/cmaj.092129.

6 Chen J, Yuan YC, Leontiadis GI, Howden CW. Recent safety concerns with proton pump inhibitors. / Clin Gastroenterol 2012:46:93-114. doi:10.1097/MCG.0b013e3182333820.

7 Lambert AA, Lam JO, Paik JJ, Ugarte-Gil C, Drummond MB, Crowell TA. Risk of community-acquired pneumonia with outpatient proton-pump inhibitor therapy: a systematic review and meta-analysis. PLoS One 2015;10:e0128004. doi:10.1371/journal.pone.0128004.

8 Barkun AN, Bardou M, Pham CQD, Martel M. Proton pump inhibitors vs. histamine 2 receptor antagonists for stress-related mucosal bleeding prophylaxis in critically ill patients: a meta-analysis. Am J Gastroenterol 2012;107:507-20, quiz 521. doi:10.1038/ajg.2011.474.

9 Sultan N, Nazareno J, Gregor J. Association between proton pump inhibitors and respiratory infections: a systematic review and meta-analysis of clinical trials. Can J Gastroenterol 2008;22:761-6. doi:10.1155/2008/821385.

10 Millett ER, Quint JK, Smeeth L, Daniel RM, Thomas SL. Incidence of community-acquired lower respiratory tract infections and pneumonia among older adults in the United Kingdom: a population-based study. PLoS One 2013;8:e75131. doi:10.1371/journal.pone.0075131.

11 Laheij RJ, Sturkenboom MC, Hassing R-J, Dieleman J, Stricker BHC, Jansen JB. Risk of community-acquired pneumonia and use of gastric acid-suppressive drugs. JAMA 2004;292:1955-60. doi:10.1001/ jama.292.16.1955.

12 Gulmez SE, Holm A, Frederiksen H, Jensen TG, Pedersen C, Hallas J. Use of proton pump inhibitors and the risk of community-acquired pneumonia: a population-based case-control study. Arch Intern Med 2007;167:950-5. doi:10.1001/archinte.167.9.950.

13 Myles PR, Hubbard RB, McKeever TM, Pogson Z, Smith CJ, Gibson JE. Risk of community-acquired pneumonia and the use of statins, ace inhibitors and gastric acid suppressants: a population-based case-control study. Pharmacoepidemiol Drug Saf 2009;18:269-75. doi:10.1002/pds.1715.

14 Sarkar M, Hennessy S, Yang Y-X. Proton-pump inhibitor use and the risk for community-acquired pneumonia. Ann Intern Med 2008;149:391-8. doi:10.7326/0003-4819-149-6-200809160-00005.

15 Dublin S, Walker RL, Jackson ML, Nelson JC, Weiss NS, Jackson LA. Use of proton pump inhibitors and $\mathrm{H} 2$ blockers and risk of pneumonia in older adults: a population-based case-control study. Pharmacoepidemiol Drug Saf2010;19:792-802. doi:10.1002/pds.1978.

16 Hermos JA, Young MM, Fonda JR, Gagnon DR, Fiore LD, Lawler EV. Risk of community-acquired pneumonia in veteran patients to whom proton pump inhibitors were dispensed. Clin Infect Dis 2012;54:3342. doi:10.1093/cid/cir767.

17 Jena AB, Sun E, Goldman DP. Confounding in the association of proton pump inhibitor use with risk of community-acquired pneumonia. J Gen Intern Med 2013;28:223-30. doi:10.1007/s11606-012-2211-5.

18 Filion KB, Chateau D, Targownik LE, et al. CNODES Investigators. Proton pump inhibitors and the risk of hospitalisation for communityacquired pneumonia: replicated cohort studies with meta-analysis. Gut 2014:63:552-8, doi:10.1136/gutjnl-2013-304738.

19 Herrett E, Gallagher AM, Bhaskaran K, et al. Data Resource Profile: Clinical Practice Research Datalink (CPRD). Int J Epidemio 2015:44:827-36. doi:10.1093/ije/dyv098.
20 Jick SS, Kaye JA, Vasilakis-Scaramozza C, et al. Validity of the general practice research database. Pharmacotherapy 2003;23:686-9. doi:10.1592/phco.23.5.686.32205.

21 Williams T, van Staa T, Puri S, Eaton S. Recent advances in the utility and use of the General Practice Research Database as an example of a UK Primary Care Data resource. Ther Adv Drug Saf 2012;3:89-99. doi:10.1177/2042098611435911.

22 Digital NHS. Hospital Episode Statistics. 2015. http://www.hscic.gov.uk/hes.

23 National Institute for Health and Care Excellence. Pneumonia in adults: diagnosis and management. NICE, 2014

24 Niederman MS. Understanding the natural history of community-acquired pneumonia resolution: vital information for optimizing duration of therapy. Clin Infect Dis 2004:39:1791-3. doi:10.1086/426031.

25 Whitaker HJ, Hocine MN, Farrington CP. The methodology of self-controlled case series studies. Stat Methods Med Res 2009;18:7-26. doi:10.1177/0962280208092342.

26 Whitaker HJ, Farrington CP, Spiessens B, Musonda P. Tutorial in biostatistics: the self-controlled case series method. Stat Med 2006;25:1768-97. doi:10.1002/sim.2302.

27 Immunisation against infectious disease Contraindications and special considerations. InSalisbury D, Ramsay M, eds. The Green Book.Public Health England, 2013: 41-8.

28 Charlson ME, Pompei P, Ales KL, MacKenzie CR. A new method of classifying prognostic comorbidity in longitudinal studies: development and validation. J Chronic Dis 1987;40:373-83. doi:10.1016/0021-9681(87)90171-8.

29 Tannen RL, Weiner MG, Xie D. Use of primary care electronic medical record database in drug efficacy research on cardiovascular outcomes: comparison of database and randomised controlled trial findings. BM/ 2009;338:b81. doi:10.1136/bmi.b81.

30 Yu M, Xie D, Wang X, Weiner MG, Tannen RL. Prior event rate ratio adjustment: numerical studies of a statistical method to address unrecognized confounding in observational studies.

Pharmacoepidemiol Drug Saf 2012;21(Suppl 2):60-8. doi:10.1002/ pds. 3235 .

31 Uddin MJ, Groenwold RH, van Staa TP, et al. Performance of prior event rate ratio adjustment method in pharmacoepidemiology: a simulation study. Pharmacoepidemiol Drug Saf 2015;24:468-77. doi:10.1002/ pds. 3724

32 Ahrens D, Behrens G, Himmel W, Kochen MM, Chenot J-F. Appropriateness of proton pump inhibitor recommendations at hospital discharge and continuation in primary care. Int J Clin Pract 2012;66:767-73. doi:10.1111/j.1742-1241.2012.02973.x.

33 Christine Bond. The over-the-counter pharmaceutical market policy and practice. Eurohealth (Lond) 2008;14(3).

34 Allison P, Waterman R. Fixed-effects negative binomial regression models. Sociol Methodol 2002:32:247-65doi:10.1111/1467-9531.00117.

35 Armstrong BG, Gasparrini A, Tobias A. Conditional Poisson models: a flexible alternative to conditional logistic case cross-over analysis. BMC Med Res Methodol 2014:14:122 doi:10.1186/1471-2288-14-122.

36 Quint JK, Müllerova H, DiSantostefano RL, et al. Validation of chronic obstructive pulmonary disease recording in the Clinical Practice Research Datalink (CPRD-GOLD). BMJ Open 2014:4:e05540. doi:10.1136/bmjopen-2014-005540.

37 Estborn L, Joelson S. Occurrence of community-acquired respiratory tract infection in patients receiving esomeprazole: retrospective analysis of adverse events in 31 clinical trials. Drug Saf 2008;31:62736. doi:10.2165/00002018-200831070-00008.

38 Estborn L, Joelson S. Frequency and time to onset of communityacquired respiratory tract infections in patients receiving esomeprazole: a retrospective analysis of patient-level data in placebo-controlled studies. Aliment Pharmacol Ther 2015;42:607-13. doi:10.1111/apt.13304

\section{Supplementary tables}

